$12-31-2016$

\title{
The Impact of MD5 File Hash Collisions On Digital Forensic Imaging
}

Gary C. Kessler

Embry-Riddle Aeronautical University

Follow this and additional works at: https://commons.erau.edu/jdfsl

Part of the Computer Engineering Commons, Computer Law Commons, Electrical and Computer Engineering Commons, Forensic Science and Technology Commons, and the Information Security Commons

\section{Recommended Citation}

Kessler, Gary C. (2016) "The Impact of MD5 File Hash Collisions On Digital Forensic Imaging," Journal of Digital Forensics, Security and Law. Vol. 11 , Article 9.

DOI: https://doi.org/10.15394/jdfsl.2016.1431

Available at: https://commons.erau.edu/jdfsl/vol11/iss4/9

This Article is brought to you for free and open access by

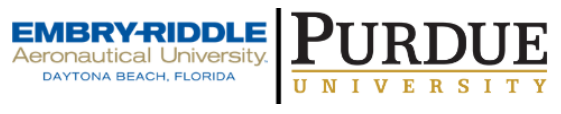
the Journals at Scholarly Commons. It has been accepted for inclusion in Journal of Digital Forensics, Security and Law by an authorized administrator of Scholarly Commons. For more information, please contact commons@erau.edu.

(c)ADFSL 


\title{
THE IMPACT OF MD5 FUE HASH COLLISIONS ON DIGITAL FORENSIC IMAGING
}

\author{
Gary C. Kessler \\ Embry-Riddle Aeronautical University \\ Daytona Beach, Florida \\ 386-226-7947 \\ gary.kessler@erau.edu
}

\begin{abstract}
The Message Digest 5 (MD5) hash is commonly used as for integrity verification in the forensic imaging process. The ability to force MD5 hash collisions has been a reality for more than a decade, although there is a general consensus that hash collisions are of minimal impact to the practice of computer forensics. This paper describes an experiment to determine the results of imaging two disks that are identical except for one file, the two versions of which have different content but otherwise occupy the same byte positions on the disk, are the same size, and have the same hash value.
\end{abstract}

Keywords: MD5 hash collisions, forensic imaging, computer forensics, digital forensics

\section{INTRODUCTION}

The use of hash functions is widely used in the practice of digital forensics to ensure the integrity of files and the accuracy of forensic imaging. The Message Digest 5 (MD5) hash algorithm remains as one of the most commonly used hashes in digital forensics (Casey, 2011; Maras, 2015; Nelson, Phillips, \& Steuart, 2015).

Hash collisions -- i.e., the occurrence where two files with different content have the same hash value -- have been identified in several well-known hash algorithms, in particular MD5 (McHugh, 2014; Wang, Feng, Lai, \& Yu, 2004; Wang \& Yu, 2005). Hashes are used for a variety of applications, including digital signature verification, computer forensic image verification, user identification and authentication, identifying known good or bad files in a hashset, and secure message exchange. The significance and meaning of a third-party being able to force hash collisions is different for these different applications; while forcing a hash collision in an authentication application could be quite serious, the impact might be less damaging when identifying files in a hashset (AccessData, 2006; Lewis, 2008; Thompson, 2005). Nevertheless, the use of hashing is so ingrained in digital forensics training and practice that the impact of such collisions in validating an evidentiary copy continues to be discussed at conferences and training sessions.

This paper will address the impact of MD5 hash collisions on validating the results of the computer forensics imaging process. Section 2 will identify the specific problem of hash collisions as it applies to imaging, followed by a restatement of the problem as a research question in Section 3. Section 4 will describe an experimental framework with which to test the research hypothesis, 
followed by test results in Section 5. Section

\section{PROBLEM STATEMENT}

The MD5 algorithm is described by Rivest (1992) and its use to validate forensic images is described in almost all computer forensics textbooks, including Casey (2011), Maras (2015), and Nelson et al. (2015). AccessData (2006) and Thompson (2005), among others, have suggested that MD5 hash collisions have minimal impact on the results of computer forensics examinations and, in practice, can be ignored. However, training in the computer forensics field for more than a quarter century has emphasized the importance of hashes as the key to proving the integrity of a digital forensic copy -- i.e., an image -- almost to the exclusion of the efficacy of training, experience, and the forensic imaging tools (Cohen, 2013).

One nightmare scenario for law enforcement, as a possible result of MD5 (or other) hash collisions, is this: A prosecutor introduces a set of $\mathrm{N}$ images of child sexual assault as evidence at trial, complete with the MD5 file hashes. The defense counters by producing a set of $\mathrm{N}$ images of the defendant on a dive boat in Aruba, complete with the same set of MD5 hashes. If this situation was possible, it can always be resolved, presumably, by viewing the original images on the evidentiary drive. However, the FUD (fear, uncertainty, and doubt) Factor has already been seeded and a good argument might then be made that could cause a jury - or jurist -- to doubt the veracity and integrity of even the original evidence because the next obvious question is: If the first set of (innocent) images has the same hash values as the second set of (damning) images, could not the second set of images have been placed on the evidentiary disk by an over-zealous prosecutor or investigator?
6 will offer some conclusions.

It is well known that MD5 hash collisions exist, although they have largely been forced to occur in the laboratory (Burr, 2006; Gutman, Naccache, \& Palmer, 2005; McHugh, 2014; Wang, Feng, Lai, \& Yu, 2004; Wang \& Yu, 2005). No one has yet reported hash collisions occurring in "nature;" that is, there are no reports of finding two different files on a given disk drive having the same MD5 hash. This is not surprising, given that there are $2^{128}$ (or $\sim 10^{43}$ ) possible MD5 hash values.

In digital forensics, we computer hash values not only on the individual files but also the entire disk that is being imaged. If we have two files, $\mathrm{A}$ and $\mathrm{B}$, that have the same hash but are of different sizes, it is clear that the image hash will be different because there will be changes not only in the file content but also in other parts of the disk, such as allocated or unallocated space. Indeed, the file system metadata -- e.g., the file size in the directory entry as well as File Allocation Table (FAT), \$Bitmap, or inode entries -- will also be different if the file sizes differ.

The impact is less obvious if files $\mathrm{A}$ and $\mathrm{B}$ are the same size because all of the file system metadata might be unchanged. Thus, is the nightmare scenario suggested above actually possible? This could theoretically only occur if one believes that the disk image hash remains the same if all of the files on the disk have the same hash. The experiment described in this paper addresses this question.

\section{RESEARCH QUESTION}

The scenario mentioned in Section 2 can be described as follows: Suppose one has two files, $\mathrm{A}$ and $\mathrm{B}$, that have different content 
but are the same size and have the same MD5 hash value. What is the effect on the hash value of two disk images that differ only in that one disk contains File A and the other disk contains File B (where Files A and B occupy the same location on the two disk images)?

The research question is to test the following null hypothesis $\left(\mathrm{H}_{0}\right)$ as follows:

- The resultant two disk images will have the same hash value.

The alternative hypothesis $\left(\mathrm{H}_{1}\right)$ is as follows:

- The resultant two disk images will have different hash values.

\section{EXPERIMENTAL SETUP}

To address the research questions, two files were needed that were the same size, had the same MD5 hash, and had different content. Selinger (2011) provides such a pair of 128byte files, called hash1.bin and hash2.bin, below:

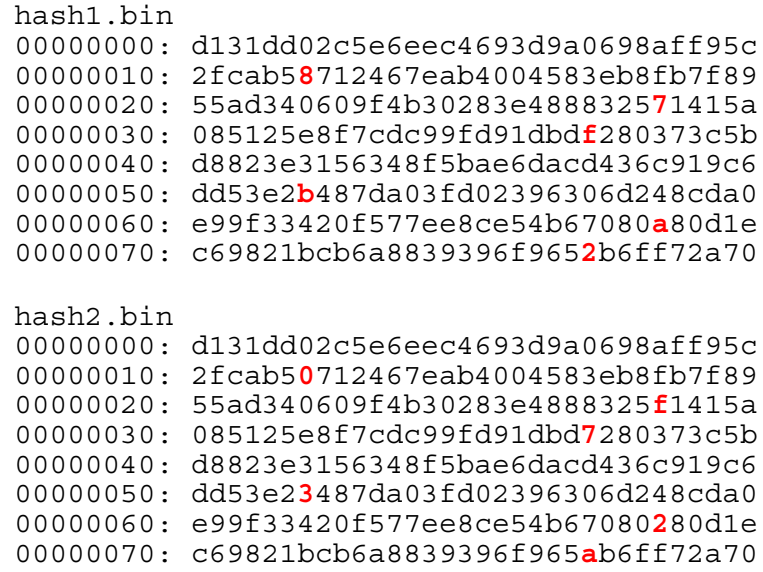

00000000: d131dd02c5e6eec4693d9a0698aff95c 00000010 : 2fcab50712467eab4004583eb8fb7f89 $00000020: 55 a d 340609 f 4 b 30283 e 4888325 f 1415 a$ 00000030 : 085125e8f7cdc99fd91dbd7280373c5b 00000040 : d8823e3156348f5bae6dacd436c919c6 00000050 : dd53e23487da03fd02396306d248cda0 00000060 : eg9f33420f577ee8ce54b67080280dle 00000070 : c69821bcb6a8839396f965ab6ff72a70

The contents of the two files differ only by six bits, shown above in the six bolded nibbles. This is confirmed when executing the $f c$ (file compare) command against the two files:

Comparing files hashl.bin and hash2.bin
$00000013: 8707$ $0000002 \mathrm{D}: 71 \mathrm{~F} 1$ $0000003 B: F 272$ $00000053:$ B4 34 $0000006 \mathrm{D}: \mathrm{A} 828$ $0000007 \mathrm{~B}: 2 \mathrm{~B} \mathrm{AB}$

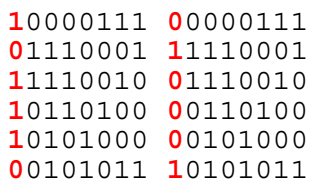

While the two files have the same 128-bit MD5 hash, it is worth noting that their 160bit Secure Hash Algorithm (SHA-1) values differ (Eastlake \& Jones, 2001). This confirms that the contents of the two files are actually different and that there is a bona fide MD5 hash collision:

File: hashl.bin

MD5 9054025255FB1A26E4BC422AEF54EB4

SHA. . A34473CF767C6108A5751A20971F1FDFBA97690A

File: hash2.bin

MD5 79054025255FB1A26E4BC422AEF54EB4

SHA 4283DD2D70AF1AD3C2D5FDC917330BF502035658

A $32 \mathrm{MB}$ thumb drive was used as the test media. Using Windows 7 , the thumb drive was formatted using the format e: /v:HASHTEST /p:1 command. This initialized a FAT16 partition where the data area was overwritten with zeroes. The contents of the thumb drive were verified using the WinHex (v18.6) hex editor. Finally, a set of seven files were copied -- six arbitrary files plus hash1.bin -- to the thumb drive. The file list and hash values were:






\section{TESTS AND RESULTS}

Four tests were conducted on the media described above. The results described in this section are summarized in Table 1.

In Test $\# 1$, the thumb drive was imaged using FTK Imager (v3.1.3.2). The purpose of this test was merely to prepare a baseline disk image and set of hash values. The image verification MD5 hash of the thumb drive was

\section{d1fdd4a0019fbedcd4459b51633ad9b8} and the complete FTK Imager report can be found in Appendix 1. The image was examined with FTK (v1.81.6) and the file listing showed the expected MD5 and SHA-1 hash values for the hash1.bin file (as shown in Section 4).

For Test \#2, the thumb drive was mounted with WinHex and the contents of hash1.bin were copied over the location where hash1.bin resided on the thumb drive (128 bytes starting at offset 0x6149). The purpose of this test was to confirm that overwriting data in this way was possible and reliable. Note that it was not necessary to change anything else on the thumb drive since the two files were the same size; no changes were necessary to the FAT table entries or to the directory name, address, or file size. The thumb drive was then reimaged. The image verification MD5 hash was

d1fdd4a0019fbedcd4459b51633ad9b8 -the same as in Test $\# 1$. This result confirms that overwriting data in this way is an adequate process and changes nothing else on the drive. A portion of the FTK Imager report can be found in Appendix 2. The FTK file listing showed the expected MD5 and SHA-1 hash values for the hash1.bin file.

For Test $\# 3$, the thumb drive was mounted in WinHex and the contents of hash2.bin were copied over the location where hash1.bin resided on the thumb drive. This test was really the crux of the hypothesis experiment since hash2.bin is the "hash-equivalent, content-different" file to hash1.bin. The thumb drive was re-imaged, yielding an image verification MD5 hash of 8045e3c1d5a44eeb5297447b85ecada4 -different than Tests $\# 1$ and $\# 2$. A portion of the FTK Imager report can be found in Appendix 3. The FTK file listing showed the expected MD5 and SHA-1 hash values for the hash2.bin file.

For Test \#4, the thumb drive was mounted with WinHex and the contents of hash1.bin were copied back over the location where hash2.bin now resided on the thumb drive. The purpose of this test was to restore the drive to its original state and confirm that Test \#3 changed nothing more than the 128 bytes where the test data resided. The fourth image verification MD5 hash was d1fdd4a0019fbedcd4459b51633ad9b8 -the same as Tests $\# 1$ and $\# 2$. This result confirms that Test \#4 had restored the disk to its initial state and that Test \#3 changed nothing more than the file data. A portion of the FTK Imager report can be found in Appendix 4. The FTK file listing showed the expected MD5 and SHA-1 hash values for the hash1.bin file.

\section{CONCLUSIONS}

The image verification MD5 hashes in Tests $\# 1, \# 2$, and $\# 4$-- images that each held the hash1.bin content -- had the same value, whereas the image verification MD5 hash value in Test $\# 3$-- when the image held the hash2.bin content -- was different from the other tests. The fact that Tests $\# 1, \# 2$, and \#4 had the same hash proved that the test process worked as desired; the fact that Test \#3 had a different result shows that the hash value of the imaged drive depends upon the actual bit content of the entire drive. Since 
the hash values of the two images are not the same, the null hypothesis $\left(\mathrm{H}_{0}\right)$ is disproven and the alternate hypothesis $\left(\mathrm{H}_{1}\right)$ is proven.

If the hash value of the disk were a function of the hashes of the individual components of the disk's contents, then one would expect to find the disk image unchanged when the files were substituted, meaning that the "nightmare scenario" could be realized. If the hash of the disk, however, were just based upon the bits on the disk, the two image hashes would be different when the files were exchanged, meaning that the scenario could not actually be perpetrated in this way.

Disproving the null hypothesis, then, is the expected result because the hash value of a disk image is supposed to be based upon the bit contents of the disk rather than the hashes of the individual files -- including file system structures and unallocated space -that compose the disk contents. Thus, even if all of the file hashes on two disks are the same, the disk image hashes will be different if the contents of the files are different.

Table 1.

Summary of the four tests and the results.

\begin{tabular}{|l|l|}
\hline Description of Test & Image MD5 Hash Value \\
\hline$\# 1$ - Drive with hash1.bin file at bytes 0x6149-0x61C8 & d1fdd4a0019fbedcd4459b51633ad9b8 \\
\hline$\# 2$ - Overwrite bytes 0x6149-0x61C8 with hash1.bin & d1fdd4a0019fbedcd4459b51633ad9b8 \\
\hline$\# 3$ - Overwrite bytes 0x6149-0x61C8 with hash2.bin & 8045e3c1d5a44eeb5297447b85ecada4 \\
\hline$\# 4$ - Overwrite bytes 0x6149-0x61C8 with hash1.bin & d1fdd4a0019fbedcd4459b51633ad9b8 \\
\hline
\end{tabular}

\section{NOTE}

All FTK Imager reports, FTK reports, and ancillary files are available for examination at

http://www.garykessler.net/gck/hash_test.zi p.

\section{AUTHOR BIOGRAPHY}

Gary C. Kessler, Ph.D., is a professor of cybersecurity and chair of the Security
Given this result, the scenario described in Section 2 cannot be realized.

It is hoped that this result will lay the concern about file hash collisions to rest as they apply to digital forensic imaging. As long as both individual files and the entire image are hashed, the theoretical occurrence of individual file collisions is not a factor in confirming the evidentiary integrity of a forensic copy.

As noted above, the SHA-1 hash values are different for the hash1.bin and hash2.bin files, although SHA-1 collisions are also theoretically possible (Stevens, Karpman, \& Peyrin, 2015; Stevens et al., 2017). Since the MD5 and SHA-1 algorithms are different, the manipulation that can create an MD5 collision cannot create a SHA-1 collision and, to date, no one has yet shown a practical method with which to cause both an MD5 and SHA-1 collision in the same file. The results of the experiment reported in this paper, however, suggests that it would not matter since a file hash collision will still result in different image file hashes. 


\section{REFERENCES}

AccessData. (2006, April). MD5 Collisions: The Effect on Computer Forensics. AccessData White Paper. Retrieved from https://adpdf.s3.amazonaws.com/papers/wp.MD5 Collisions.en_us.pdf

Burr, W. (2006, March/April). Cryptographic hash standards: Where do we go from here? IEEE Security \& Privacy, 4(2), 88-91. Retrieved from http://www.csee.wvu.edu/\%7Ekaterina/ Teaching/CS-465-Spring2007/HashStandards.pdf

Casey, E. (2011). Digital Evidence and Computer Crime, 3rd ed. Amsterdam: Elsevier.

Cohen, F. (2013). Digital Forensic Evidence Examination, 5th ed. Livermore (CA): Fred Cohen \& Associates. Retrieved from http://all.net/books/2013-DFE-

Examination.pdf

Eastlake, D., 3rd, \& Jones, P. (2001, September). US Secure Hash Algorithm 1 (SHA1). Requests for Comments (RFC) 3174. Retrieved from https://www.rfceditor.org/rfc/rfc3174.txt

Gutman, P., Naccache, D., \& Palmer, C.C. (2005, May/June). When hashes collide. IEEE Security \& Privacy, 3(3), 68-71. Retrieved

from https://researchspace.auckland.ac.nz/bits tream/handle /2292/269/269.pdf

Lewis, D.L. (2008, December 1). The Hash Algorithm Dilemma -- Hash Value Collisions. Forensic Magazine. Retrieved from http://www.forensicmag.com/article/200 8/12/hash-algorithmdilemma\%E2\%80\%93hash-value-collisions

Maras, M. H. (2015). Computer Forensics: Cybercriminals, Laws, and Evidence, 2nd ed. Burlington, MA: Jones \& Bartlett Learning.

McHugh, N. (2014, October 31). How I created two images with the same MD5 hash. Retrieved from http://natmchugh.blogspot.com/2014/10 /how-i-created-two-images-with-samemd5.html

Nelson, B., Phillips, A., \& Steuart, C. (2015). Guide to Computer Forensics and Investigations, 5th ed. Boston: Course Technology.

Rivest, R. (1992, April). The MD5 Message Digest Algorithm. Request for Comments (RFC) 1321. Retrieved from https://www.rfceditor.org/rfc/rfc1321.txt 
Selinger, P. (2011, October 11). MD5 Collision Demo. Retrieved from http://www.mathstat.dal.ca $/ \sim$ selinger $/$ md5collision/

Stevens, M., Bursztein, E., Karpman, P., Albertini, A., Markov, Y., Bianco, A.P., \& Biasse, C. (2017, February 23). Announcing the first SHA1 collision. Google Security Blog. Retrieved from https://security.googleblog.com/2017/02/ announcing-first-sha1-collision.html

Stevens, M., Karpman, P., \& Peyrin, T. (2015, October 8). Freestart collision on full SHA-1. Cryptology ePrint Archive, Report 2015/967. Retrieved from https://eprint.iacr.org/2015/967.pdf

Thompson, E. (2005, February). MD5 collisions and the impact on computer forensics. Digital Investigation, 2(1), 3640.

Wang, X., Feng, D., Lai, X., \& Yu, H. (2004, August). Collisions for Hash Functions MD4, MD5, HAVAL-128 and RIPEMD. Retrieved from http://eprint.iacr.org/2004/199.pdf

Wang, X.Y. \& Yu, H.B. (2005, May). How to break MD5 and other hash functions. Advances in Cryptology-Eurocrypt'05, pp. 19-35. Retrieved from merlot.usc.edu/csac-f06/papers /Wang05a.pdf 


\section{APPENDICES}

\section{Appendix 1: FTK Imager report for Test \#1}

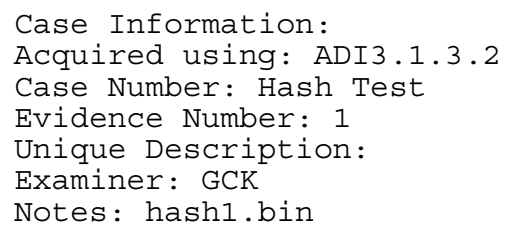

Appendix 2: FTK Imager report (partial) for Test \#2

Created By AccessData ${ }^{\circledR}$ FTK $^{\circledR}$ Imager 3.1.3.2

Case Number: Hash Test 2

Evidence Number: 2

Examiner: GCK

Notes: hash1.bin (overwritten)

Information for $C: \backslash U$ sers $\backslash g c k \backslash$ Desktop $\backslash$ hash_test $\backslash$ Test2:

[Computed Hashes]

MD5 checksum: difdd4a0019fbedcd4459b51633ad9b8 


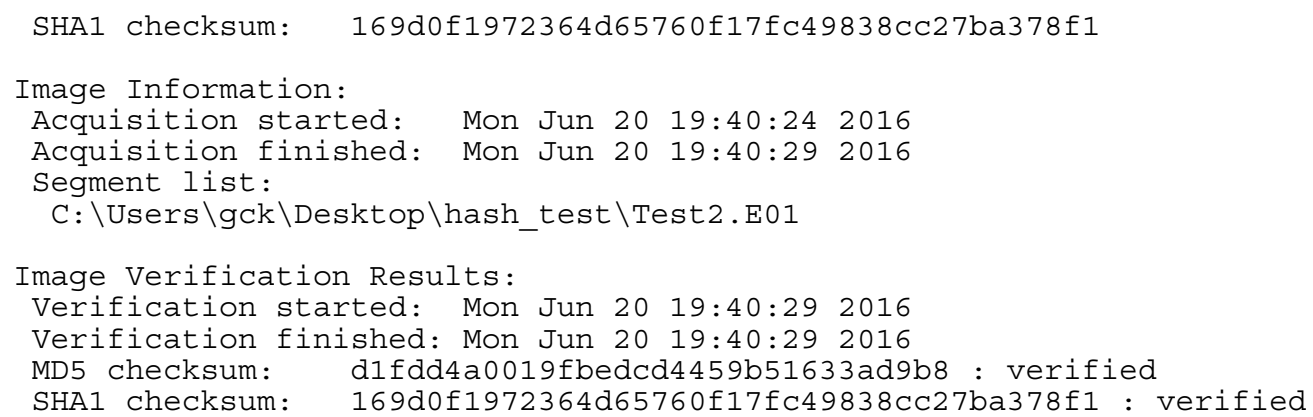

\section{Appendix 3: FTK Imager report (partial) for Test \#3}

Created By AccessData ${ }^{\circledR}$ FTK $^{\circledR}$ Imager 3.1.3.2

Case Number: Hash Test

Evidence Number: 3

Examiner: GCK

Notes: hash2.bin overwrite

Information for $C: \backslash U$ sers $\backslash g c k \backslash$ Desktop $\backslash$ hash_test $\backslash$ Test3:

[Computed Hashes]

MD5 checksum: $8045 e 3 c 1 d 5 a 44$ eeb5297447b85ecada4

SHA1 checksum: 177774 eefa63b5e67c04a2e9d2d875e2353400df

Image Information:

Acquisition started: Mon Jun 20 19:43:14 2016

Acquisition finished: Mon Jun 20 19:43:18 2016

Segment list:

C: \Users \gck \Desktop \hash test \Test3.E01

Image Verification Results:

Verification started: Mon Jun 20 19:43:18 2016

Verification finished: Mon Jun 20 19:43:18 2016

MD5 checksum: 8045e3c1d5a44eeb5297447b85ecada4 : verified

SHA1 checksum: 177774eefa63b5e67c04a2e9d2d875e2353400df : verified

\section{Appendix 4: FTK Imager report (partial) for Test \#4}

Created By AccessData ${ }^{\circledR}$ FTK $^{\circledR}$ Imager 3.1.3.2

Case Number: Hash Test

Evidence Number: 4

Examiner: GCK

Notes: hashl.bin re-written

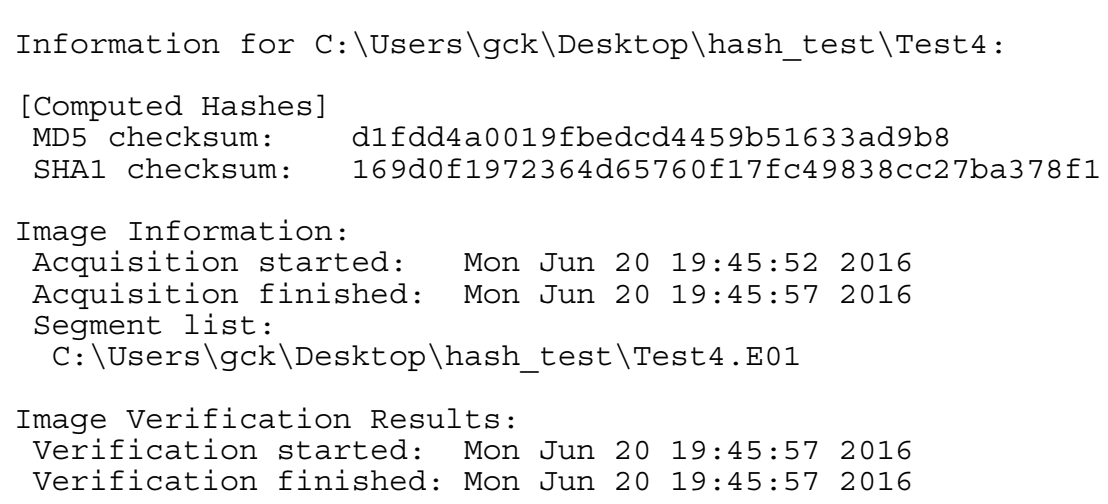


MD5 checksum: dlfdd4a0019fbedcd4459b51633ad9b8 : verified

SHA1 checksum: 169d0f1972364d65760f17fc49838cc27ba378f1 : verified 